\title{
Eco-Epidemiological Model with Fatal Disease in the Prey
}

\author{
David Greenhalgh ${ }^{1}$, Qamar J. A. Khan ${ }^{2}$ and Fatma Ahmed \\ Al-Kharousi ${ }^{3}$ \\ ${ }^{1}$ Department of Mathematics and Statistics, University of Strathclyde, \\ Livingstone Tower, 26 Richmond Street, Glasgow G1 1XH, UK. \\ ${ }^{2 \& 3}$ Department of Mathematics and Statistics, Sultan Qaboos University, \\ P.O. Box 36, PC. 123 Alkhoud Muscat, Sultanate of Oman, \\ david.greenhalgh@strath.ac.uk, qjalil@squ.edu.om, \\ fatma.ahmed.rus@cas.edu.om
}

\begin{abstract}
We investigate a model consisting of a predator population and both susceptible and infected prey populations. The predator can feed on either prey species but instead of choosing individuals at random the predator feeds preferentially on the most abundant prey species. More specifically we assume that the likelihood of a predator catching a susceptible prey or an infected prey is proportional to the numbers of these two different types of prey species. This phenomenon, involving changing preference from susceptible to infected prey, is called switching. Mukhopadhyay studied a switching model and proposed that the interaction of predators with infected prey is beneficial for the growth of the predator. In this model, we assume that the predator will eventually die as a result of eating infected prey. We find a threshold parameter $R_{0}$ and showed that the disease will be eradicated from the system if $R_{0}<1$.
\end{abstract}

\section{Introduction}

The two fields of epidemiology and ecology were studied separately for decades. Kermack and McKendrick were among the first to apply mathematical modelling to study the spread of diseases. Meanwhile, Lotka and Volterra were simultaneously pioneering the study of the interaction of biological species. In the late nineteen eighties and the early nineteen nineties, these two fields intermixed and a new hybrid area of research emerged called eco-epidemiology. Since its formation, a large amount of work has been done considering the intersection of epidemiological and ecological problems [[1]-[15]]. Although these two areas 
can be viewed as independent, they have many overlapping features.

An example of this overlap can be vividly seen in the Salton Sea, which is located in the desert of Southern California and has no outlet. In the summer the weather reaches 128 degrees Fahrenheit and the water evaporates very quickly, leaving the salt behind. This process increases the salinity of the Salton Sea and decreases oxygen levels as it is more difficult for salt water to bind oxygen than fresh water. The Salton Sea is the main stop for many species of migratory birds such as pelicans, but thousands of thousands of water birds, most of which were pelicans, and fish have died. The actual cause of this is still unknown, but the evidence increasingly points towards toxic algal blooms. Many fish have died due to deoxygenation. Agricultural waste has been deposited in the Salton Sea for nearly a century. The resulting increased concentration of phosphorus and nitrogen on the one hand makes the sea water highly nutritious and able to support rapid fish growth but on the other hand has severe adverse effects as it causes algae to bloom and produce toxins. The algal bloom grows and dies very quickly and in doing so it pulls oxygen from the sea water. The shortage of oxygen in the tissues of the infected fish causes botulism outbreaks to occur and the later stages of the disease cause infected fish to rise to near the surface of the water to seek oxygen [7]. This bacterial botulism infection in the tissues of the infected fish passes from one fish to another and as the numbers of infected fish increase the scale of the epidemic increases exponentially and consequently massive mortality occurs in the fish population. Pelicans prey upon and eat these vulnerable fish whilst the fish are still alive. The pelicans thus ingest botulism toxins and develop avian botulism, a debilitating neurological disease, and die off in huge numbers [13].

Chattopadhyay and Bairagi [7] and Sarkar et al. [11] studied mathematical models for the interaction of fish (Tilapia) and birds (pelicans). In both models the authors assumed that the predators (the pelicans) did not consume the susceptible prey (the Tilapia). Later, Greenhalgh and Haque [9] described a ratio-dependent predator-prey interaction model with a similar assumption where the susceptible class experienced no predation; this is unlikely to be realistic for most species. Bairagi et al. [14] modified Greenhalgh and Haque's model [7] by introducing the interaction of the pelicans with susceptible fish and proposed that feeding on infected fish increased the death rate of the pelicans.

In this paper we study a model in which both susceptible and infected prey populations are exposed to the predator, with varying degrees of exposure. However, the predator feeds preferentially on the most numerous prey type. This implies a kind of switching from the susceptible class to the infected class, and vice versa, as these two types of prey change in numerical superiority. Switching may simply come about due to individual behaviour changing with varying abundance of that class of prey. We also suggest that that infected Tilapia suffer a higher predation risk than susceptible ones. This is because the death struggle of the infected Tilapia causes them to come closer to the surface of the sea gulping for air and thus they become more attractive prey for fish eating birds.

There are several examples in the literature where a predator preferentially 
preys upon the prey species which is most abundant at any given time [[16]-[19]]. Mathematical models have been proposed to to describe this sort of switching. Such models generally involve interaction of a single predator species with two prey species [[20]-[28]].

In this paper we investigate a model consisting of a predator population, (pelicans), and both susceptible and infected prey populations, (Tilapia). The predator can feed on either prey species but instead of choosing individuals at random the predator feeds preferentially on the most abundant prey species, a sort of switching. More specifically, we assume that the chance of a predator catching either a susceptible prey or an infected prey is proportional to the numbers of these two different types of prey species. Mukhopadhyay and Bhattacharyya [6] studied a switching model and concluded that the interaction of pelicans with infected Tilapia is beneficial for the growth of the pelicans. In contrast we suppose that eating infected fish is fatal for the pelicans and, hence, this interaction is detrimental to the pelican population. One of the main aims for the study of eco-epidemiological models is to find a condition for which the eco-system becomes disease free. We find a basic reproductive number $R_{0}$ of infection which determines whether the disease will die out. The disease will die out from the eco-system if $R_{0}<1$.

\section{The Model Formulation}

This investigation utilizes an eco-epidemiological model which describes the interaction between the susceptible and diseased fish population (Tilapia) and their predators (pelicans) in the Salton Sea. We assume that the disease is transmitted by direct contact with the prey. Due to the presence of the disease, the prey population is divided into two disjoint classes, the susceptible fish population, $S$ per unit designated area, and the infected fish population, $I$ per unit designated area. Therefore, at time $t$ the total Tilapia population is $N(t)=S(t)+I(t)$. The predator population per unit designated area is denoted by $Y$. If there is no predation and no infection then the fish population follows a logistic growth model. Infected fish do not recover and due to the fact that infected fish die very quickly we assume that infected fish do not reproduce. The pelicans are most likely to consume the type of prey, susceptible prey or infected prey, which has the largest numbers. Both susceptible and infected Tilapia are preyed upon by pelicans, but the pelicans preferentially eat the infected fish, because the infection causes the fish to be more vulnerable to predation. Infected Tilapia experience deoxygenation in the body due to the infection and, as the disease progresses, come to the surface of the lake seeking oxygen. As a result of the disease, these infected Tilapia become more attractive to pelicans. We assume that the birds have no food source other than fish and that the toxicity level is so high that eating infected fish does more harm than good. We also presuppose that the death rate of the pelicans is proportional to the ingestion of sick fish [12] rather than a constant.

From the above assumptions, we formulate the following model: 


$$
\begin{aligned}
& \frac{d S}{d t}=r S\left(1-\frac{S+I}{K}\right)-\lambda S I-\frac{p S^{2} Y}{S+\alpha I}, \\
& \frac{d I}{d t}=\lambda S I-\frac{c I^{2} Y}{S+\alpha I}-\gamma I, \\
& \frac{d Y}{d t}=\frac{\delta_{1} p S^{2} Y}{S+\alpha I}-\frac{\delta_{2} c I^{2} Y}{S+\alpha I}-d Y .
\end{aligned}
$$

The term

$$
\frac{p S^{2} Y}{S+\alpha I},
$$

is not defined at $S=I=0$. However as for both $S$ and $I$ small

$$
0<\frac{S}{S+\alpha I} \leq 1
$$

it is natural to interpret $(2.2)$ as zero at $S=I=0$. Similarly we interpret

$$
\frac{c I^{2} Y}{S+\alpha I}
$$

as zero at $S=I=0$.

The parameters now have the following interpretations:

- $r$ : The species growth rate of Tilapia in the breeding subpopulation per day;

- $\gamma$ : The per capita death rate of infected prey per day;

- $p$ and $c$ : The pelican response rate towards susceptible and infected Tilapia multiplied by the area under consideration, measured in units of area per day;

- $\alpha$ : The preference of the predator, i.e. the predator will prey upon the infected prey $\alpha$ times more than on the uninfected prey;

- $K$ : The carrying capacity of the environment for the prey in the absence of disease per unit area;

- $\delta_{1}$ and $\delta_{2}$ : The rate of conversion to predator of susceptible and infected prey respectively per day; 
- $\lambda$ : The rate of transmission multiplied by the area under consideration measured in units of area per day; and

- $d$ : The death rate of the predators per day.

Note that $\lambda, p$ and $c$ are measured in units of area per day because $S, I$ and $Y$ are taken as densities per unit area. If $S, I$ and $Y$ are taken as numbers $\lambda, p$ and $c$ are rates, per unit time, and in their definitions there is no multiplication factor for the area under consideration.

We assume that all the parameters in the model are positive, and that $S(0) \geq$ $0, I(0) \geq 0$ and $Y(0) \geq 0$. As the right-hand side of equations (2.1) are Lipschitz continuous in $D=\{(S, I, Y): S, I, Y \geq 0\}$, thus it is straightforward to show that these equations have a unique solution in $D$ [30].

\section{Basic Reproduction Number and Equilibrium Analysis}

A key parameter of the system is the basic reproduction number of the disease in the prey, $R_{0}$, defined by

$$
R_{0}=\frac{\lambda S^{*}}{\gamma} .
$$

Here, $\lambda$ is the rate of infection multiplied by the area under consideration and $\lambda S^{*}$ is the infection rate of new infectives appearing in a totally susceptible prey population where $S^{*}=K$ and $\gamma$ is the death rate of infected prey because of natural death and disease induced mortality and $\frac{1}{\gamma}$ is the duration of infection of an infected prey. So $\frac{\lambda S^{*}}{\gamma}$ is the disease reproduction number in the prey at $S^{*}=K$.

We examine the equilibrium of system (2.1). We obtain equilibrium solutions by setting time derivatives to zero.

Theorem 3.1 In system (2.1) $\bar{S}, \bar{I}$ and $\bar{Y}$ are the equilibrium values of $S, I$ and $Y$ respectively, we found five equilibrium points:

(i) $\bar{E}_{0}=[0,0,0]$.

If the disease is highly infectious and fatal, then all uninfected prey becomes infected and will die because disease is fatal. After interacting with fatally diseased prey the population of predators will become extinct. So the population is extinct and this equilibrium always exists.

(ii) $\bar{E}_{1}=[\bar{S}, 0, \bar{Y}]$, where

$$
\bar{S}=\frac{d}{\delta_{1} p}, \quad \text { and } \quad \bar{Y}=\frac{r}{p}\left(1-\frac{d}{K \delta_{1} p}\right)=\frac{r}{p}\left(\frac{K-\bar{S}}{K}\right) .
$$

So this equilibrium where there is no infected prey will always exist. 
(iii) $\bar{E}_{2}=\left[\frac{\gamma}{\lambda}, \frac{r(K \lambda-\gamma)}{\lambda(K \lambda+r)}, 0\right]$.

If there is no predator and disease is not highly infectious, then prey population survive as uninfected and infected prey. This equilibrium where there are no predators will exist if the carrying capacity per unit area times the area being considered times the disease transmission rate is greater than or equal to the death rate of infected prey.

(iv) $\bar{E}_{3}=[K, 0,0]$.

This equilibrium corresponds to the situation where the infectious prey population, and the predator population has gone to extinction and the susceptible population has gone to its carrying capacity. It is always possible.

(v) $\bar{E}_{4}=[\bar{S}, \bar{I}, \bar{Y}]$.

This is a possible equilibrium where susceptible prey, infected prey and predators co-exist. It will exist and be unique if and only if

$$
\lambda K>\gamma, \delta_{1} p \gamma>d \lambda, \text { and } \frac{r+K \lambda}{K \lambda-\gamma} \frac{\alpha d}{r}+\frac{\delta_{2} c}{\lambda}>\frac{(r+K \lambda)^{2}}{(K \lambda-\gamma)^{2}} \frac{\gamma}{r^{2}}\left(\frac{\delta_{1} p \gamma}{\lambda}-d\right) .
$$

If the above conditions are satisfied, then

$$
\bar{S}=\frac{d(\bar{x}+\alpha) \bar{x}}{\delta_{1} p \bar{x}^{2}-\delta_{2} c}, \bar{I}=\frac{d(\bar{x}+\alpha)}{\delta_{1} p \bar{x}^{2}-\delta_{2} c} \text { and } \bar{Y}=\frac{(\bar{x}+\alpha)(\lambda \bar{S}-\gamma)}{c} .
$$

Here $\bar{x}$ is the unique possible root of the cubic equation

$$
f(x)=a_{0} x^{3}+a_{1} x^{2}+a_{2} x+a_{3}=0,
$$

where

and

$$
\begin{aligned}
& a_{0}=K p\left(d \lambda-\delta_{1} p \gamma\right) \\
& a_{1}=p d \alpha K \lambda-r c \delta_{1} K p+r d c \\
& a_{2}=p \delta_{2} c \gamma K+r d \alpha c+(r+\lambda K) d c \\
& a_{3}=r K c^{2} \delta_{2}+(r+\lambda K) d \alpha c
\end{aligned}
$$

Define

$$
\Delta=f(\alpha) f(\beta)=\frac{4 a_{2}^{3}}{27 a_{0}}-\frac{a_{1}^{2} a_{2}^{2}}{27 a_{0}^{2}}+\frac{4 a_{1}^{3} a_{3}}{27 a_{0}^{2}}-\frac{2 a_{1} a_{2} a_{3}}{3 a_{0}}+a_{3}^{2},
$$

where $\alpha$ and $\beta$ are the turning points of (3.4). 


\section{Proof.}

$$
\begin{aligned}
f(\alpha)= & a_{0} \alpha^{3}+a_{1} \alpha^{2}+a_{2} \alpha+a_{3}, \\
f(\beta)= & a_{0} \beta^{3}+a_{1} \beta^{2}+a_{2} \beta+a_{3}, \\
\Delta= & f(\alpha) f(\beta), \\
= & a_{0}^{2}(\alpha \beta)^{3}+a_{0} a_{1}(\alpha \beta)^{2}(\alpha+\beta)+a_{0} a_{2}(\alpha \beta)\left(\alpha^{2}+\beta^{2}\right)+a_{0} a_{3}\left(\alpha^{3}+\beta^{3}\right) \\
& +a_{1}^{2}(\alpha \beta)^{2}+a_{1} a_{2}(\alpha \beta)(\alpha+\beta)+a_{1} a_{3}\left(\alpha^{2}+\beta^{2}\right)+a_{2}^{2}(\alpha \beta) \\
& +a_{2} a_{3}(\alpha+\beta)+a_{3}^{2} .
\end{aligned}
$$

Here

$$
\begin{aligned}
& \left(\alpha^{3}+\beta^{3}\right)=(\alpha+\beta)^{3}-3(\alpha \beta)(\alpha+\beta), \\
& \left(\alpha^{2}+\beta^{2}\right)=(\alpha+\beta)^{2}-2(\alpha \beta) .
\end{aligned}
$$

We write

$$
f^{\prime}(x)=3 a_{0} x^{2}+2 a_{1} x+a_{2},
$$

where

$$
\begin{aligned}
\alpha \beta & =\frac{a_{2}}{3 a_{0}}, \\
\text { and } \quad \alpha+\beta & =-\frac{2 a_{1}}{3 a_{0}},
\end{aligned}
$$

substituting (3.8), (3.9), (3.11) and (3.12) in (3.7) and after algebraic simplification, we obtain

$$
\Delta=\frac{4 a_{2}^{3}}{27 a_{0}}-\frac{a_{1}^{2} a_{2}^{2}}{27 a_{0}^{2}}+\frac{4 a_{1}^{3} a_{3}}{27 a_{0}^{2}}-\frac{2 a_{1} a_{2} a_{3}}{3 a_{0}}+a_{3}^{2} .
$$

There are three cases:

1. There are no feasible co-existence equilibria if
(a) $\gamma>\lambda K$,
(b) (i) $\delta_{1} p K>d>\delta_{1} p \frac{\gamma}{\lambda}$ and (ii) $a_{1}>0$, or (iii) $d \geq \delta_{1} p K$,

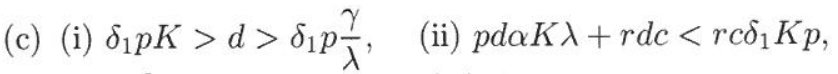
(iii) $a_{1}^{2}>3 a_{0} a_{2}$ and (iv) $\Delta>0$,

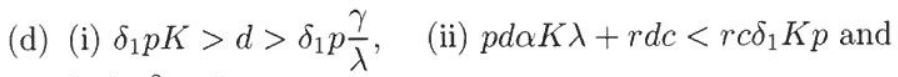 (iii) $a_{1}^{2}<3 a_{0} a_{2}$. 
2. There are two distinct feasible co-existence equilibria if

(i) $K \lambda>\gamma$, (ii) $\delta_{1} p K>d>\delta_{1} p \frac{\gamma}{\lambda}$, (iii) $p d \alpha K \lambda+r d c<r c \delta_{1} K p$,

(iv) $a_{1}^{2}>3 a_{0} a_{2}$ and (v) $\Delta<0$. In this case the equation (3.4) has two distinct strictly positive real roots $\bar{x}_{1}$ and $\bar{x}_{2}$.

3. There is a unique feasible co-existence equilibrium

(a) If (i) $K \lambda>\gamma$, (ii) $p d \alpha K \lambda+r d c<r c \delta_{1} K p$, (iii) $a_{1}^{2}>3 a_{0} a_{2}$ and (iv) $\Delta=0$, then the equation (3.4) has a unique positive real root $\bar{x}$ and the equations (3.3) give a unique feasible co-existence equilibrium.

(b) If (i) $K \lambda>\gamma$ and (ii) $\delta_{1} p \gamma>d \lambda$, then the equation (3.4) has a unique positive real root $\bar{x}$ and the equations (3.3) give a unique feasible co-existence equilibrium, if and only if

$$
\frac{r+K \lambda}{K \lambda-\gamma} \frac{\alpha d}{r}+\delta_{2} \frac{c}{\lambda}>\frac{(r+K \lambda)^{2}}{(K \lambda-\gamma)^{2}} \frac{\gamma}{r^{2}}\left(\delta_{1} \frac{p \gamma}{\lambda}-d\right) .
$$

In this case the equation (3.4) has a unique positive real root $\bar{x}$, and the equations (3.3) give a unique feasible co-existence equilibrium.

Proof. The proof of the assertions concerning the first four equilibria are obvious. To prove the fifth, suppose that $\bar{E}_{4}$, exists with $\bar{S}, \bar{I}$ and $\bar{Y}$ all positive and define $\bar{x}=\frac{\bar{S}}{I}>0$. Then from the equilibrium equations we can see that (3.3) holds also.

$$
\bar{Y}=\frac{\bar{S}+\alpha I}{p \bar{S}}\left[r\left(1-\frac{\bar{S}+\bar{I}}{K}\right)-\lambda \bar{I}\right] .
$$

Equating the two values for $\bar{Y}$ in (3.3) and (3.13) and substituting for $\bar{S}$ and $\bar{I}$ in terms of $\bar{x}$ we obtain that $\bar{x}$ satisfies the cubic equation (3.4). From the expression for $\bar{Y}>0$, we require that

$$
K>\bar{S}>\frac{\gamma}{\lambda}
$$

Hence if

1. (a) $\gamma>\lambda K$, there are no feasible co-existence equilibria, because $\bar{Y}>0$ and $\bar{S} \leq K$.

(b) If (i) $\delta_{1} p K>d>\delta_{1} p \frac{\gamma}{\lambda}$ and (ii) $a_{1}>0$, or (iii) $d \geq \delta_{1} p K$, then $a_{0} \geq 0, a_{1} \geq 0, a_{2}>0, a_{3}>0$, so equation (3.4) has no real positive roots and there are no feasible co-existence equilibria.

(c) If (i) $\delta_{1} p K>d>\delta_{1} p \frac{\gamma}{\lambda}$, (ii) $p d \alpha K \lambda+r d c<r c \delta_{1} K p$, (iii) $a_{1}^{2}>3 a_{0} a_{2}$ and (iv) $\Delta>0$, then $f(x)$ has two real positive turning points $0<$ $\alpha_{1}<\beta_{1}$ and $\alpha_{1}$ is a maximum, $\beta_{1}$ is a minimum, but $f\left(\alpha_{1}\right) f\left(\beta_{1}\right)=$ $\Delta>0$ so $f\left(\beta_{1}\right)>0$ and $f(x)>0$ for $x \geq 0$, so (3.4) has no real positive roots and again there are no co-existence equilibria. 
(d) Note that, $f^{\prime}(x)=3 a_{0} x^{2}+2 a_{1} x+a_{2}$. Hence if (i) $\delta_{1} p K>d>\delta_{1} p \frac{\gamma}{\lambda}$, (ii) $p d \alpha K \lambda+r d c<r c \delta_{1} K p$ and (iii) $a_{1}^{2} \leq 3 a_{0} a_{2}$, then $f(x)$ has no real turning points or has a single point of inflexion point so it is monotonic increasing and $f(0)=a_{3}>0$ so again equation (3.4) has no real positive roots and there are no feasible co-existence equilibria.

2. If (i) $K \lambda>\gamma$, (ii) $\delta_{1} p K>d>\delta_{1} p \frac{\gamma}{\lambda}$, (iii) $p d \alpha K \lambda+r d c<r c \delta_{1} K p$, (iv) $a_{1}^{2}>3 a_{0} a_{2}$ and (v) $\Delta<0$, then the function $f(x)$ has two distinct real positive turning points $0<\alpha_{1}<\beta_{1}$ with $f\left(\alpha_{1}\right) f\left(\beta_{1}\right)<0$. Hence $f\left(\alpha_{1}\right)>0>f\left(\beta_{1}\right)$ and the equation (3.4) has two distinct real positive roots, one is $\bar{x}_{1}$ in $\left(\alpha_{1}, \beta_{1}\right)$ and the other is $\bar{x}_{2}$ in $(\beta, \infty)$. Each root corresponds to a distinct feasible co-existence equilibrium. Hence in this case there are two distinct co-existence equilibria.

3. (a) If (i) $K \lambda>\gamma$, (ii) $p d \alpha K \lambda+r d c<r c \delta_{1} K p$, (iii) $a_{1}^{2}>3 a_{0} a_{2}$ and (iv) $\Delta=0$, then a similar argument to (2) shows that $f\left(\beta_{1}\right)=0$ and equation (3.4) has unique positive root $\bar{x}$. Note that $f(x)$ can be written as

$$
\begin{aligned}
f(\bar{x}) & =\left(p K \bar{x}+\frac{r c}{\lambda}\right)\left[\bar{x}^{2}\left(d \lambda-\delta_{1} p \gamma\right)+\alpha d \lambda \bar{x}+\delta_{2} c \gamma\right] \\
& +(r+K \lambda) d c(\alpha+\bar{x})-r c\left(K-\frac{\gamma}{\lambda}\right)\left(\bar{x}^{2} \delta_{1} p-\delta_{2} c\right)=0 .
\end{aligned}
$$

So we deduce that

$$
\bar{x}^{2} \delta_{1} p-\delta_{2} c>0
$$

or

$$
\bar{x}>\bar{x}_{0}=\frac{\delta_{2} c}{\delta_{1} p} .
$$

Moreover, $\bar{S}>\frac{\gamma}{\lambda}$, if and only if

$$
g(\bar{x})=x^{2}\left(d \lambda-\delta_{1} p \gamma\right)+(\alpha d \lambda) \bar{x}+\delta_{2} c \gamma>0,
$$

which is always true here. So in this case (3.4) gives a unique feasible co-existence equilibrium.

(b) If (i) $K>\frac{\gamma}{\lambda}$ and (ii) $\delta_{1} p \gamma>d \lambda$, then $a_{0}<0, \quad a_{2}$ and $a_{3}>0$ so by Descartes' rule of signs and the fact that $f(0)=a_{3}>0$ and $\lim _{x \rightarrow \infty} f(x)=-\infty$ then $f(x)=0$ has a unique positive root $\bar{x}$. By using the same argument as in 3(a) we deduce that

$$
\bar{x}>\bar{x}_{0}=\frac{\delta_{2} c}{\delta_{1} p},
$$

and $\bar{S}>\frac{\gamma}{\lambda}$, if and only if $g(\bar{x})>0$. As $\delta_{1} p \gamma>d \lambda, g(\bar{x})>0$ is equivalent to $\bar{x}<\bar{x}_{2}$, where $\bar{x}_{2}$ is the unique positive root of $g(\bar{x})>0$. Also using the equilibrium equation $f(\bar{x})=0$,

$$
-r c h(\bar{x})=p \lambda K \bar{x} g(\bar{x})+(r+K \lambda) \lambda d c(\alpha+\bar{x}),
$$


where

$$
h(\bar{x})=x^{2}\left(d \lambda-\delta_{1} p \gamma\right)+(\alpha d \lambda) x+\delta_{2} c \gamma<0,
$$

which implies $K>\bar{S}$.

Therefore, $\bar{x}<\bar{x}_{2}$ if and only if $f\left(\bar{x}_{2}\right)<0$. Hence $\bar{x}<\bar{x}_{2}$ is equivalent to

$$
(r+K \lambda)\left(\alpha+\bar{x}_{2}\right)<\left(K-\frac{\gamma}{\lambda}\right)\left(\bar{x}_{2}^{2} \delta_{1} p-\delta_{2} c\right),
$$

as $g\left(\bar{x}_{2}\right)=0$, which is the same as

$$
\bar{x}_{2}^{2} \delta_{1} p-\delta_{2} c=\frac{d \lambda\left(\bar{x}_{2}^{2}+\alpha \bar{x}_{2}\right)}{\gamma},
$$

using (3.18) and (3.19) we obtain

$$
\bar{x}_{2}>\frac{\gamma}{r} \frac{(r+K \lambda)}{(K \lambda-\gamma)}=\bar{x}_{1} .
$$

But $\bar{x}_{2}>\bar{x}_{1}$ is equivalent to $g\left(\bar{x}_{1}\right)>0$ which gives

$$
\frac{\alpha d}{r} \frac{(r+K \lambda)}{(K \lambda-\gamma) r}+\delta_{2} \frac{c}{\lambda}>\left(\delta_{1} p-d\right) \frac{\gamma}{r^{2}}\left(\frac{r+K \lambda}{K \lambda-\gamma}\right)^{2} .
$$

\section{Boundedness Results}

Proposition 4.1 The susceptible prey and infected prey are always bounded above.

Proof. On adding the first and second equations of the system (2.1) we obtain

$$
\frac{d S}{d t}+\frac{d I}{d t} \leq \frac{r}{\lambda} S\left(1-\frac{S+I}{K}\right)
$$

It follows that $\lim \sup _{t \rightarrow \infty}(S+I) \leq K$. Hence $\limsup _{t \rightarrow \infty} S(t) \leq K$ and $\limsup \sup _{t \rightarrow \infty} I(t) \leq K$. This completes the proof of Proposition 4.1.

Proposition 4.2 The trajectories of system (2.1) are bounded.

Proof. Define the function $l=S+I+Y$ and take the time derivative along the solution of (2.1).

$$
\frac{d l}{d t}=\frac{d S}{d t}+\frac{d I}{d t}+\frac{d Y}{d t} .
$$


Now for any positive constant $q$,

$$
\begin{aligned}
\frac{d l}{d t}+q l & =r S-r\left(\frac{S+I}{K}\right)-\frac{p S^{2} Y}{S+\alpha I}-\frac{c I^{2} Y}{S+\alpha I}-\gamma I \\
& +\frac{\delta_{1} p S^{2} Y}{S+\alpha I}-\frac{\delta_{2} c I^{2} Y}{S+\alpha I}-d Y+q S+q I+q Y \\
& =(r+q) S+(q-\gamma) I-(1-\delta) \frac{p S^{2} Y}{S+\alpha I} \\
& +\left(q-\frac{c I^{2}}{S+\alpha I}-\frac{\delta_{2} c I^{2}}{S+\alpha I}-d\right) Y-r\left(\frac{S+I}{K}\right) \\
& \leq(r+q) S+(q-\gamma) I+\left(q-\frac{c I^{2}}{S+\alpha I}-\frac{\delta_{2} c I^{2}}{S+\alpha I}-d\right) Y \\
& \leq(r+q) S+(q-d) Y+(q-\gamma) I .
\end{aligned}
$$

Choose $q$ to be the minimum of $\{d, \gamma\}$. By Proposition 4.1 there exists $t_{0}$ such that for $t \geq t_{0}, S \leq 2 K$. Hence for $t \geq t_{0}$

$$
\frac{d l}{d t}+q l \leq m=2 K\left(\frac{r}{\lambda}+q\right) .
$$

This implies that for $t \geq t_{0}$

$$
l \leq \frac{m}{q}+l\left(t_{0}\right) e^{-q\left(t-t_{0}\right)},
$$

from which we can deduce that

$$
\limsup _{t \rightarrow \infty} l \leq \frac{m}{q}
$$

independently of the initial conditions. This completes the proof of Proposition 4.2 .

Corollary 4.3 If $\epsilon>0$, then the region

$$
X=\left\{0 \leq S \leq 2 K, I \geq 0, Y \geq 0, S+I+Y \leq \frac{m}{q}+\epsilon\right\}
$$

is an invariant region for (2.1).

Proof. This is straightforward from the proof of Proposition 4.2. 


\section{Stability}

We now examine the local and global asymptotic stability of the system about the equilibria found in Section (3.3). The stability matrix or Jacobian of the system $(2.1)$ around a general equilibrium point $[\bar{S}, \bar{I}, \bar{Y}]$ is

$$
\mathbf{J}=\left(\begin{array}{lll}
J_{11} & J_{12} & J_{13} \\
J_{21} & J_{22} & J_{23} \\
J_{31} & J_{32} & J_{33}
\end{array}\right)
$$

where

$$
\begin{aligned}
& J_{1 \mp}-2 \frac{r \bar{S}}{K}-\frac{r \bar{I}}{K}-\bar{I} \lambda-2 \frac{p \bar{S} \bar{Y}}{\bar{S}+\alpha \bar{I}}+\frac{p \bar{S}^{2} \bar{Y}}{(\bar{S}+\alpha \bar{I})^{2}}, \\
& J_{1 \mp}=\frac{r \bar{S}}{K}-\lambda \bar{S}+\frac{p \alpha \bar{S}^{2} \bar{Y}}{(\bar{S}+\alpha \bar{I})^{2}}, \quad J_{13}=-\frac{p \bar{S}^{2}}{\bar{S}+\alpha \bar{I}}, \\
& J_{2 \mp} \bar{I}+\frac{c \bar{I}^{2} \bar{Y}}{(\bar{S}+\alpha \bar{I})^{2}}, J_{22}=\lambda \bar{S}-2 \frac{c \bar{I} \bar{Y}}{\bar{S}+\alpha \bar{I}}+\frac{c \alpha \bar{I}^{2} \bar{Y}}{(\bar{S}+\alpha \bar{I})^{2}}-\gamma, \\
& J_{25}=\frac{c \bar{I}^{2}}{\bar{S}+\alpha \bar{I}}, J_{31}=2 \frac{\delta_{1} p \bar{S} \bar{Y}}{\bar{S}+\alpha \bar{I}}-\frac{\delta_{1} p \bar{S}^{2} \bar{Y}}{(\bar{S}+\alpha \bar{I})^{2}}+\frac{\delta_{2} c \bar{I}^{2} \bar{Y}}{(\bar{S}+\alpha \bar{I})^{2}}, \\
& J_{3 \bar{z}=} \frac{\delta_{1} p \alpha \bar{S}^{2} \bar{Y}}{(\bar{S}+\alpha \bar{I})^{2}}-2 \frac{\delta_{2} c \bar{I} \bar{Y}}{\bar{S}+\alpha \bar{I}}+\frac{\delta_{2} c \alpha \bar{I}^{2} \bar{Y}}{(\bar{S}+\alpha \bar{I})^{2}}, \quad \text { and } \\
& \mathcal{J}_{33} \frac{\delta_{1} p \bar{S}^{2}}{\bar{S}+\alpha \bar{I}}-\frac{\delta_{2} c \bar{I}^{2}}{\bar{S}+\alpha \bar{I}}-d .
\end{aligned}
$$

\subsection{Dynamics of the System around $\bar{E}_{0}=[0,0,0]$.}

The Jacobian matrix is not well-defined at the zero equilibrium point $\bar{E}_{0}$. Recall that to show that $\bar{E}_{0}$ is unstable it is sufficient to show that given a small neighbourhood $U$ of $\bar{E}_{0}$ there is at least one trajectory starting in $U$ that does not tend to $\bar{E}_{0}$.

\section{Proposition 5.1}

(i) If $S(0)>0$ then no trajectories can approach the origin for large times.

(ii) If $S(0)=0$ then all trajectories will approach the origin for large times.

Hence $\bar{E}_{0}$ is stable.

\section{Proof.}


(i) From the first equation of (2.1), we get

$$
\frac{d}{d t}(\ln S)=r\left(1-\frac{S+I}{K}\right)-\lambda I-\frac{p S Y}{S+\alpha I} .
$$

If $S \rightarrow 0, I \rightarrow 0$ and $Y \rightarrow 0$, then

$$
\frac{d}{d t}(\ln S) \rightarrow r
$$

Hence there is a small ball $B$ with centre $\bar{E}_{0}$ and radius $\epsilon$ such that within this ball

$$
\frac{d}{d t}(\ln S) \geq \frac{\epsilon}{2}
$$

If $(S, I, Y) \rightarrow(0,0,0)$, then as $t \rightarrow \infty$ there exists $t_{0}$ such that $(S, I, Y) \in$ $B$ for $t \geq t_{0}$. Moreover from (5.1)

$$
S\left(t_{0}\right)=S(0) \exp \left[\int_{0}^{t_{0}}\left\{r\left(1-\frac{S+I}{K}\right)-\lambda I-\frac{p S Y}{S+\alpha I}\right\} d \xi\right]>0 .
$$

Hence using (5.2) for $t \geq t_{0}, S(t) \geq S\left(t_{0}\right) e^{\frac{\epsilon}{2}\left(t-t_{0}\right)}$. So $S(t)$ cannot approach zero for large times. This is a contradiction. Thus (i) is true.

(ii) If $S(0)=0$ then $S(t)=0$ for all $t$.

(a) If $I(0)=0$ and $Y(0)=0$, then from the second equation of $(2.1), I(t)=0$ for all time, and from the third equation of $(2.1), Y(t)=0$ for all time. Hence the result of (ii) is true in this case.

(b) If $I(0)>0$ and $Y(0)=0$, then again $Y(t)=0$ for all time, and from the second equation of $(2.1)$

$$
\frac{d I}{d t}=-\gamma I
$$

So $I(t) \rightarrow 0$ as $t \rightarrow \infty$. Hence the result of (ii) is true in this case.

(c) If $I(0)>0$ and $Y(0)>0$, then from the second and third equations of (2.1)

$$
\frac{d I}{d t} \leq-\gamma I \text { and } \frac{d Y}{d t} \leq-d Y
$$

Therefore $I(t)$ and $Y(t) \rightarrow 0$ as $t \rightarrow \infty$ and the result of (ii) is also true in this case. This completes the proof of Proposition 5.1. 
(d) If $I(0)=0, Y(0)>0$, then $\frac{d I}{d t}=0$, i.e. $I(t)=0$ for all time, and from the third equation of $(2.1)$

$$
\frac{d Y}{d t}=-d Y
$$

Therefore $Y(t) \rightarrow 0$ as $t \rightarrow \infty$ and the result (ii) is true in this case.

\subsection{Stability of the System around $\bar{E}_{1}$}

We now examine the local asymptotic stability of the second equilibrium point

$$
\bar{E}_{1}=\left[\bar{S}_{1}, 0, \bar{Y}_{1}\right]=\left[\frac{d}{\delta_{1} p}, 0, \frac{r}{p}\left(1-\frac{d}{K \delta_{1} p}\right)\right],
$$

where the infected prey have gone extinct. The stability matrix is

$$
\mathbf{J}^{(1)}=\left(\begin{array}{ccc}
-\frac{r \bar{S}_{1}}{K} & -\frac{r \bar{S}_{1}}{K}-\lambda \bar{S}_{1}+p \alpha \bar{Y} & -p \bar{S}_{1} \\
0 & \lambda \bar{S}_{1}-\gamma & 0 \\
\delta_{1} p \bar{Y}_{1} & -\delta_{1} p \alpha \bar{Y}_{1} & 0
\end{array}\right) .
$$

One eigenvalue is $\omega=\lambda \bar{S}_{1}-\gamma$. The other two satisfy the equation

$$
\omega^{2}+\frac{r \bar{S}_{1}}{K} \omega+p^{2} \delta_{1} \bar{S}_{1} \bar{Y}_{1}=0 .
$$

By the Routh-Hurwitz conditions [21] equation (5.3) has only roots with negative real parts. Hence this equilibrium is locally stable if $\bar{S}_{1}<\frac{\gamma}{\lambda}$ (i.e. $d<\frac{\gamma p \delta_{1}}{\lambda}$ ) and unstable if $\bar{S}_{1}>\frac{\gamma}{\lambda}$ (i.e. $d>\frac{\gamma p \delta_{1}}{\lambda}$ ).

\subsection{Stability of the System around $\bar{E}_{2}$.}

We now turn to the third equilibrium point

$$
\bar{E}_{2}=\left[\bar{S}_{2}, \bar{I}_{2}, 0\right]=\left[\frac{\gamma}{\lambda}, \frac{r}{\lambda} \frac{\lambda K-\gamma}{\lambda K+r}, 0\right],
$$

where there is no predator only susceptible and infected prey. In this case the stability matrix is: 


$$
\mathbf{J}^{(2)}=\left(\begin{array}{ccc}
-\frac{r \gamma}{K \lambda} & -\left(\frac{r}{K}+\lambda\right) \frac{\gamma}{\lambda} & -\frac{p \bar{S}_{2}^{2}}{\bar{S}_{2}+\alpha \bar{I}_{2}} \\
\lambda \bar{I}_{2} & 0 & -\frac{c \bar{I}_{2}^{2}}{\bar{S}_{2}+\alpha \bar{I}_{2}} \\
0 & 0 & J_{33}^{(2)}
\end{array}\right),
$$

where $J_{33}^{(2)}=\frac{\delta_{1} p \bar{S}_{2}^{2}}{\bar{S}_{2}+\alpha \bar{I}_{2}}-\frac{\delta_{2} c \bar{I}_{2}^{2}}{\bar{S}_{2}+\alpha \bar{I}_{2}}-d$.

One eigenvalue is $J_{33}^{(2)}$. The other eigenvalues satisfy the equation

$$
\omega^{2}+\frac{r \gamma}{K \lambda} \omega+\left(\frac{r}{K}+\lambda\right) \gamma \bar{I}_{2}=0,
$$

so again have strictly negative real parts. Hence $\bar{E}_{2}$ is locally asymptotically stable if $J_{33}^{(2)}<0$ and unstable if $J_{33}^{(2)}>0$. Substituting in the equilibrium values for $\bar{S}_{2}$ and $\bar{I}_{2}$ from (5.4) we see that the condition for stability $J_{33}^{(2)}<0$ is equivalent to

$$
\left(d-\frac{\delta_{1} p \gamma}{\lambda}\right) \frac{\gamma}{r^{2}} \frac{(r+\lambda K)^{2}}{(\lambda K-\gamma)^{2}}+\frac{\alpha d}{r} \frac{r+\lambda K}{\lambda K-\gamma}+\frac{\delta_{2} c}{\lambda}>0,
$$

which is the third inequality of (3.2).

\subsection{Stability of the System around $\bar{E}_{3}$.}

Next we look at the fourth equilibrium point

$$
\bar{E}_{3}=\left[\bar{S}_{3}, 0,0\right]=[K, 0,0],
$$

where the infected prey and the predator have gone extinct. The stability matrix about this equilibrium is

$$
\mathbf{J}^{(3)}=\left(\begin{array}{ccc}
-r & -r-\lambda K & -p K \\
0 & \lambda K-\gamma & 0 \\
0 & 0 & \delta_{1} p K-d
\end{array}\right) .
$$

The eigenvalues are clearly $-r, \lambda K-\gamma$ and $\delta_{1} p K-d$. Hence this equilibrium is locally asymptotically stable if

$$
K<\min \left\{\frac{\gamma}{\lambda}, \frac{d}{\delta_{1} p}\right\}
$$

otherwise it is unstable. 


\subsection{Stability of the System around $\bar{E}_{4}$.}

The Jacobian for the system at $\bar{E}_{4}$ is

$$
\left(\begin{array}{ccc}
-\frac{r \bar{S}}{K}-\frac{\bar{S} \bar{Y} p}{A}+\frac{p \bar{S}^{2} \bar{Y}}{A^{2}} & -\frac{r \bar{S}}{K}-\lambda \bar{S}+\frac{p \bar{S}^{2} \bar{Y} \alpha}{A^{2}} & -\frac{p \bar{S}^{2}}{A} \\
\lambda \bar{I}+\frac{c \bar{I}^{2} \bar{Y}}{A^{2}} & -\frac{c \bar{I} \bar{Y}}{A}+\frac{c \bar{I}^{2} \bar{Y} \alpha}{A^{2}} & \frac{-\bar{I}^{2} c}{A} \\
\frac{2 \bar{S} \bar{Y} \delta_{1} p-d \bar{Y}}{A} & \frac{-2 \delta_{2} c \bar{I} \bar{Y}-\alpha d \bar{Y}}{A} & 0
\end{array}\right),
$$

where

$$
A=\bar{S}+\alpha \bar{I}
$$

Let

$$
\begin{aligned}
P & =-\frac{r \bar{S}}{K}-\frac{\bar{S} \bar{Y} p \alpha \bar{I}}{A^{2}}<0 ; \\
L & =\frac{-p \bar{S}^{2}}{A}<0 ; \\
M & =\lambda \bar{I}+\frac{c \bar{I}^{2} \bar{Y}}{A^{2}}>0 ; \\
N & =\frac{-c \bar{I} \bar{Y}}{A}+\frac{c \bar{I}^{2} \bar{Y} \alpha}{A^{2}}=-\frac{c \bar{I} \bar{Y} \bar{S}}{A^{2}}<0 ; \\
R & =\frac{-\bar{I}^{2} c}{A}<0 ; \\
U & =\frac{-2 \delta_{2} c \bar{I} \bar{Y}-\alpha d \bar{Y}}{A}<0 ; \\
Q & =-\frac{r \bar{S}}{K}-\lambda \bar{S}+\frac{p \bar{S}^{2} \bar{Y} \alpha}{A^{2}}, \\
& =\bar{S}\left(-\frac{r}{K}-\lambda+\frac{p \bar{S} \bar{Y} \alpha}{(\bar{S}+\alpha \bar{I})^{2}}\right),
\end{aligned}
$$

where

$$
\frac{p \bar{S} \bar{Y} \alpha}{(\bar{S}+\alpha \bar{I})^{2}}<1
$$

or

$$
p \bar{S} \bar{Y} \alpha<\bar{S}^{2}+2 \bar{S} \bar{I} \alpha+\alpha^{2} \bar{I}^{2},
$$

which is true because $p<2$. Lafferty and Morris [29] experimentally found that the predation rate of piscivorous birds on infected fish is, on average, 31 times higher than the predation rate on uninfected fish. On this ground Bairagi et al. [8] assume that the predator consumes infected prey only and interaction with uninfected fish is zero. $\bar{Y}<\bar{I}$, because at endemic equilibrium there are more infected fish than pelicans i.e. at equilibrium the infected fish population will 
be higher than pelicans population. Hence $Q<0$.

$$
T=\frac{\bar{Y}}{A}\left(2 \bar{S} \delta_{1} p-d\right)>0
$$

where

$$
\frac{\delta_{1} p \bar{S}^{2}}{A}-\frac{\delta_{2} c \bar{I}^{2}}{A}=d,
$$

since $\bar{x}$ will be unique positive root of $f(x)=0$ if

$$
\delta_{1} p \gamma>d \lambda,
$$

where

$$
\bar{S}>\frac{\gamma}{\lambda} .
$$

The Jacobian can be written as

$$
\left(\begin{array}{ccc}
P & Q & L \\
M & N & R \\
T & U & 0
\end{array}\right) .
$$

This leads to the characteristic equation

$\xi^{3}-\xi^{2}(P+N)+\xi(P N-U R-Q M-L T)+(P U R-Q R T-L M U+L N T)=0$.

The characteristic equation can be written as

$$
\xi^{3}+B_{1} \xi^{2}+B_{2} \xi+B_{3}=0,
$$

where

$$
\begin{aligned}
& B_{1}=-(P+N)>0 \\
& B_{2}=P N-U R-Q M-L T \\
& B_{3}=P U R-Q R T-L M U+L N T .
\end{aligned}
$$

Now

$$
B_{1} B_{2}>B_{3},
$$

implies that

$$
-(P+N)(P N-U R-Q M-L T)>P U R-Q R T-L M U+L N T,
$$

or

$$
-P^{2} N+P Q M+P L T-P N^{2}+N U R+N Q M+Q R T+L M U>0,
$$


where

$$
\begin{array}{r}
-P^{2} N-P N^{2}=-P N(P+N)>0, P Q M>0, \\
P L T>0, N Q M>0, Q R T>0, L M U>0, \\
N U R+L M U=U(N R+L M) \text { where, } U<0 .
\end{array}
$$

So

$$
\begin{aligned}
N R+L M & =\frac{1}{A^{3}}\left[c^{2} \bar{I}^{3} \bar{Y} \bar{S}-p \bar{S}^{2}\left(A^{2} \bar{I} \lambda+c \bar{I}^{2} \bar{Y}\right)\right], \\
& =\frac{\bar{S} \bar{I}}{A^{3}}\left[c^{2} \bar{I}^{2} \bar{Y}-p \bar{S}\left(A^{2} \lambda+c \bar{I} \bar{Y}\right)\right], \\
& =\frac{\bar{S} \bar{I}}{A^{3}}\left[c \bar{I} \bar{Y}(c \bar{I}-p \bar{S})-p \bar{S} A^{2} \lambda\right],
\end{aligned}
$$

and

$$
c \bar{I}-p \bar{S}<0 \quad \text { if } \quad c \bar{I}<p \bar{S},
$$

so if

$$
\bar{x}>\frac{c}{p},
$$

then

$$
N U R+L M U>0
$$

Thus

$$
B_{1} B_{2}>B_{3} \text { if } \bar{x}>\frac{c}{p} .
$$

Using equation (3.14), $\bar{x}>\frac{c}{p}$ if and only if

$$
\begin{aligned}
(r+\lambda K)\left[\frac{c^{2}}{p^{2}}\left(d \lambda-\delta_{1} p \gamma\right)+\alpha d \lambda \frac{c}{p}+\delta_{2} c \gamma\right] & +d \lambda(r+\lambda K)\left(\alpha+\frac{c}{p}\right) \\
& >r(\lambda K-\gamma)\left(\frac{\delta_{1} c^{2}}{p}-\delta_{2} c\right) .
\end{aligned}
$$

This is a necessary and sufficient condition for $\bar{x}>\frac{c}{p}$.

Theorem 5.2 If the equilibrium point $\bar{x}$ exists, $B_{3}>0$, and inequality (5.13) is true, then it will be asymptotically stable.

\section{Numerical Solution}

The system (2.1) has been integrated numerically using a Runge-Kutta-Fehlberg fourth-fifth order method. Table 1 contains a representative set of values used for the hypothetical parameters in the simulation. For initial data, a slight perturbation of the equilibrium values was used. $\alpha, r, \delta_{1}, \delta_{2}$ and $\gamma$ have a units 
Table 1: Representative set of parameter values used for model equation (2.1).

\begin{tabular}{|c|c|c|c|c|c|c|c|c|c|}
\hline$p$ & $\alpha$ & $r$ & $c$ & $k$ & $\delta_{1}$ & $\delta_{2}$ & $\gamma$ & $\lambda$ & \\
\hline 0.9 & 6 & 1.5 & 3 & 50 & 0.1 & 0.01 & 0.2 & 1 & \\
\hline
\end{tabular}

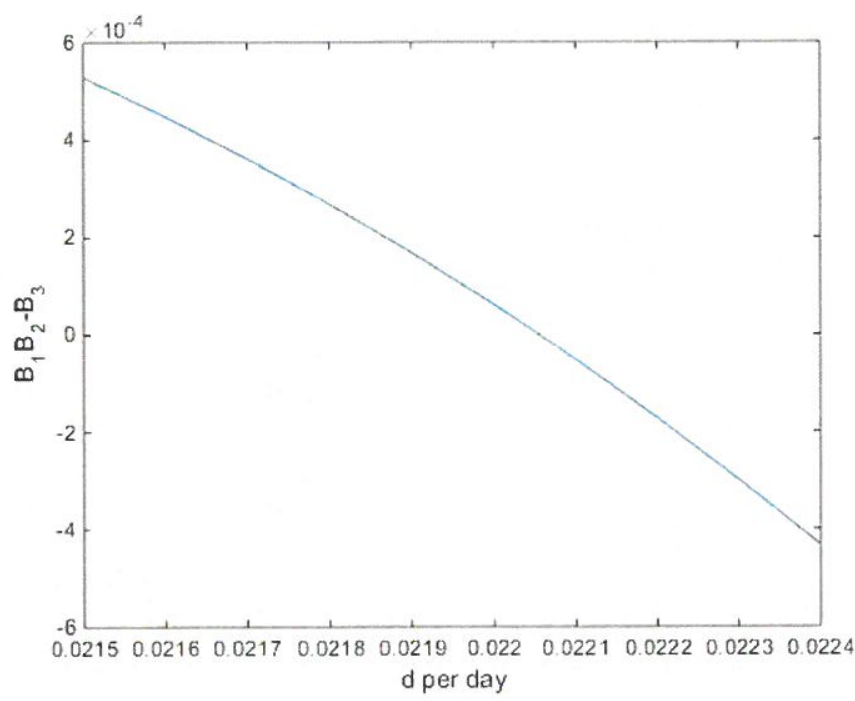

Figure 1: Plot of $B_{1} B_{2}-B_{3}$ as a function of the parameter $d$.

of "per day", $p, c$ and $\lambda$ are measured in units of "area per day", while $K, S, I$ and $Y$ have units of "number per unit area". The numerical results show that there is a Hopf bifurcation for this system where stable behaviour changes to unstable as the parameter $d$ is varied. This bifurcation point is approximately when $d=0.02206$ per day as illustrated in Figure 1 which contains a plot of $B_{1} B_{2}-B_{3}$ as a function of the parameter $d$.

Figures 2, 3 and 4 show a stable solution for the system when $d=0.022$ per day, while Figures 5, 6 and 7 show an unstable solution when the value $d=0.0221$ per day is used.

There is another numerical solution we obtain with different values of parameters, where the system is asymptotically stable.

$\alpha, r, k, \delta_{1}, \delta_{2}, d$ and $\gamma$ have units of "per day", $p, c$ and $\lambda$ are measured in units of "area per day" while $K, S, I$ and $Y$ have units of "number per unit area". 


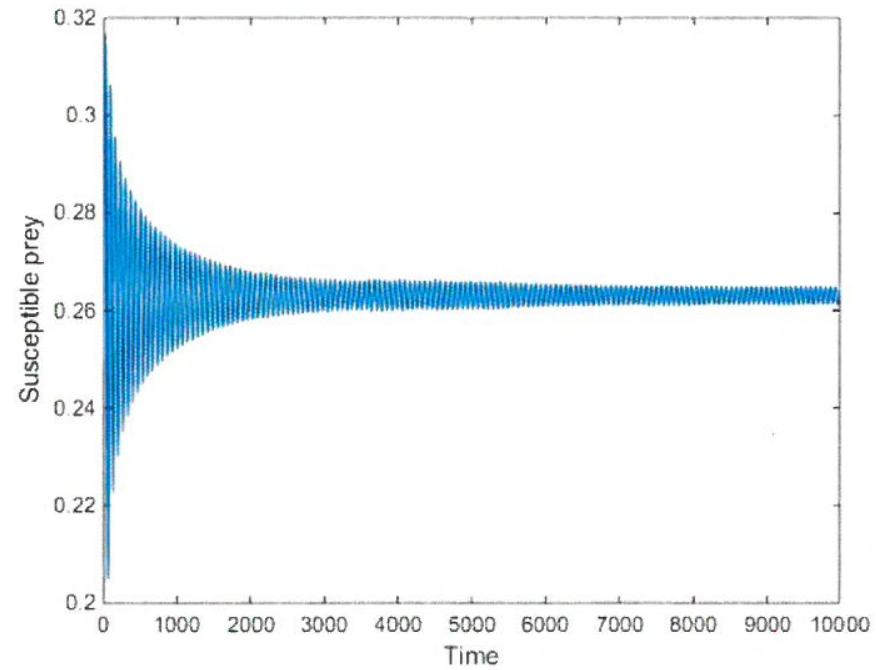

Figure 2: Hopf bifurcation of susceptible prey with respect to $d$ when $d=0.022$ per day.

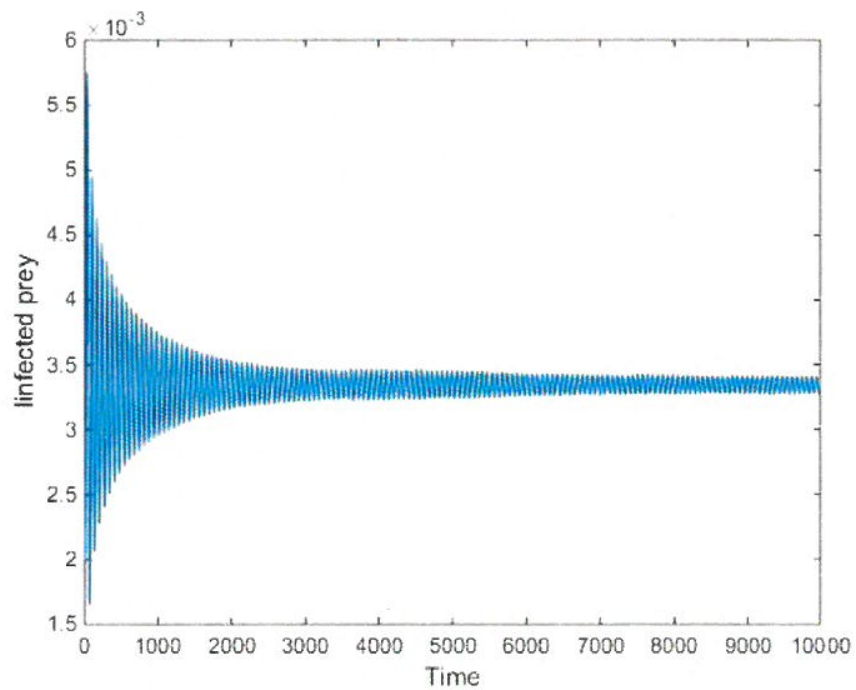

Figure 3: Hopf bifurcation of infected prey with respect to $d$ when $d=0.022$ per day. 


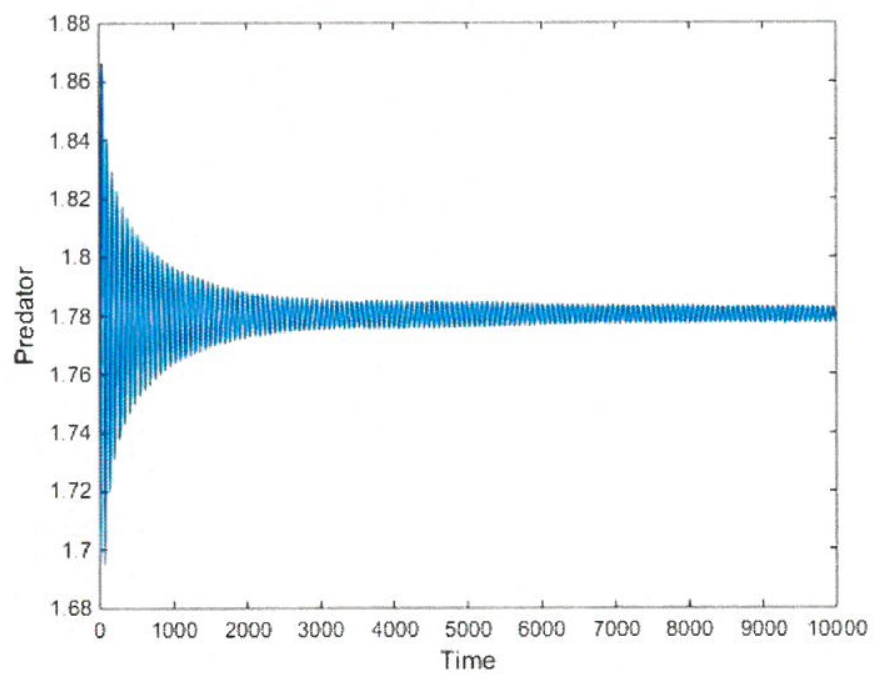

Figure 4: Hopf bifurcation of predator with respect to $d$ when $d=0.022$ per day.

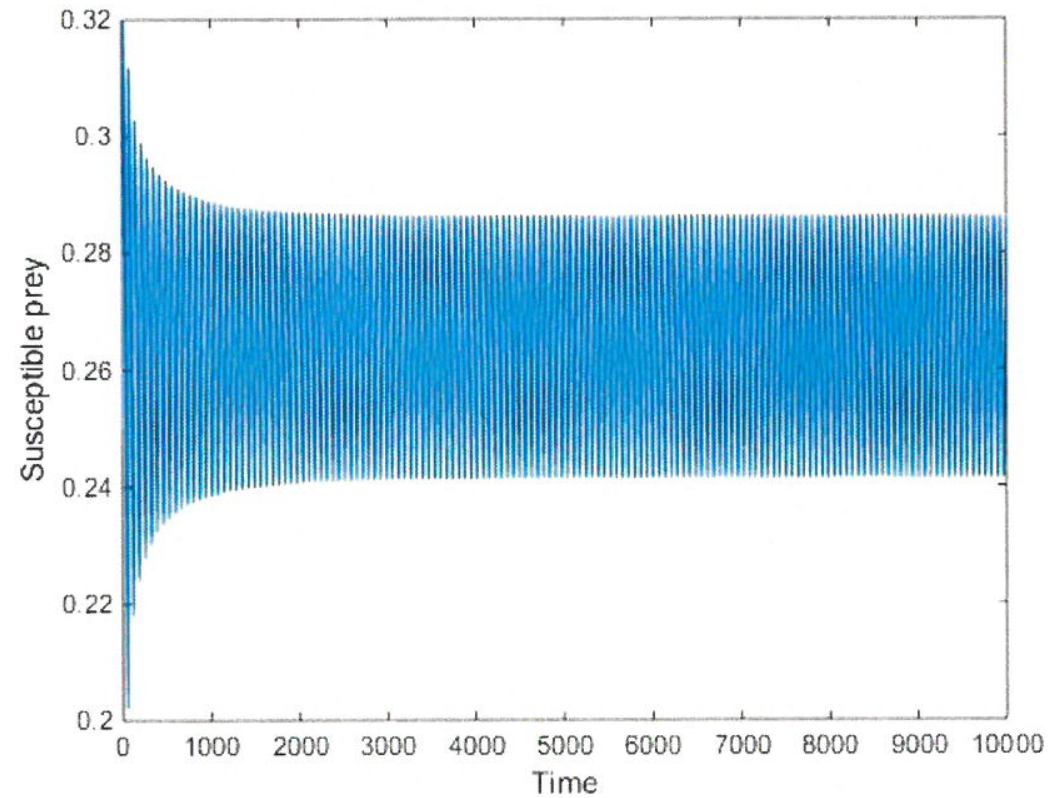

Figure 5: Hopf bifurcation of susceptible prey with respect to $d$ when $d=0.0221$ per day. 


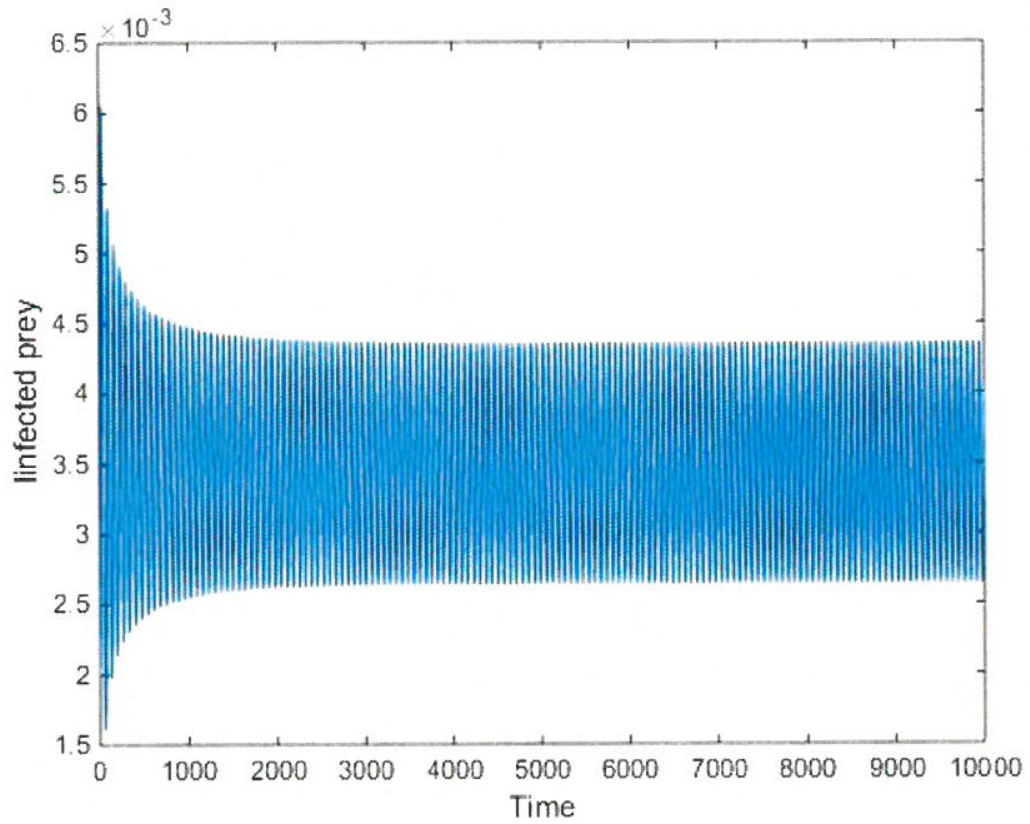

Figure 6: Hopf bifurcation of infected prey with respect to $d$ when $d=0.0221$ per day.

Table 2: Representative set of parameter values used for model equation (2.1).

\begin{tabular}{|c|c|c|c|c|c|c|c|c|c|}
\hline$p$ & $\alpha$ & $r$ & $c$ & $k$ & $\delta_{1}$ & $\delta_{2}$ & $d$ & $\gamma$ & $\lambda$ \\
\hline 0.34 & 0.1 & 3.5 & 2.2 & 50 & 0.9 & 0.02 & 1.1 & 0.02 & 1 \\
\hline
\end{tabular}




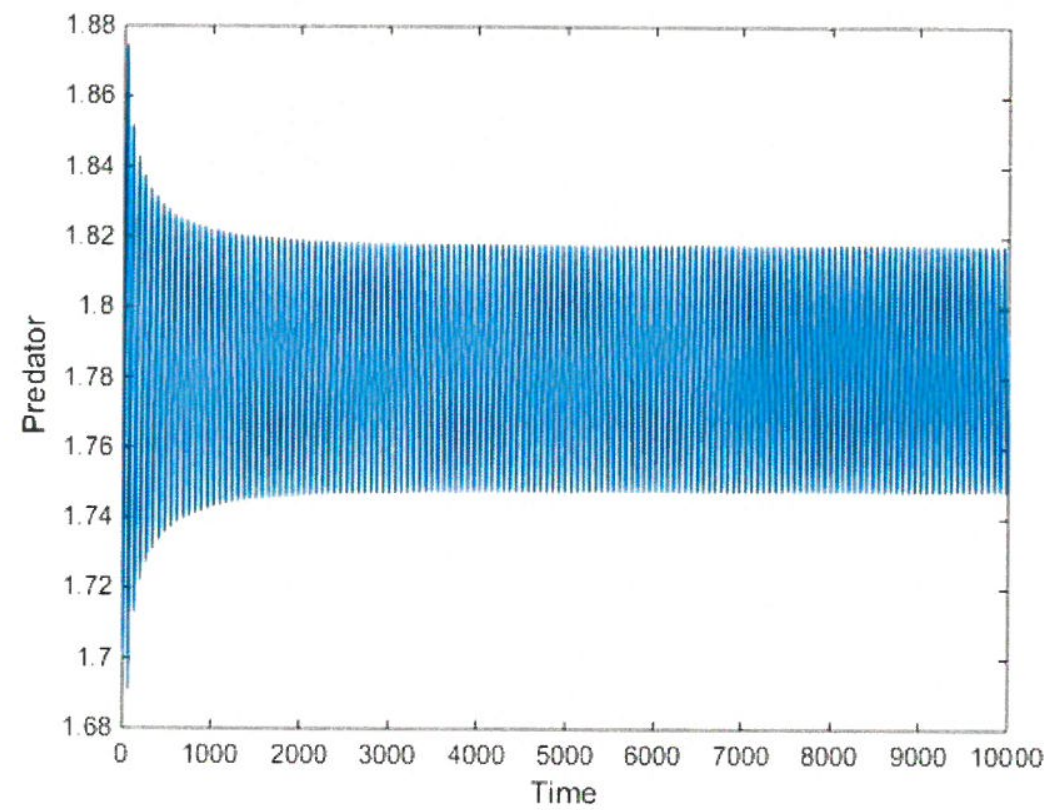

Figure 7: Hopf bifurcation of predator with respect to $d$ when $d=0.0221$ per day.

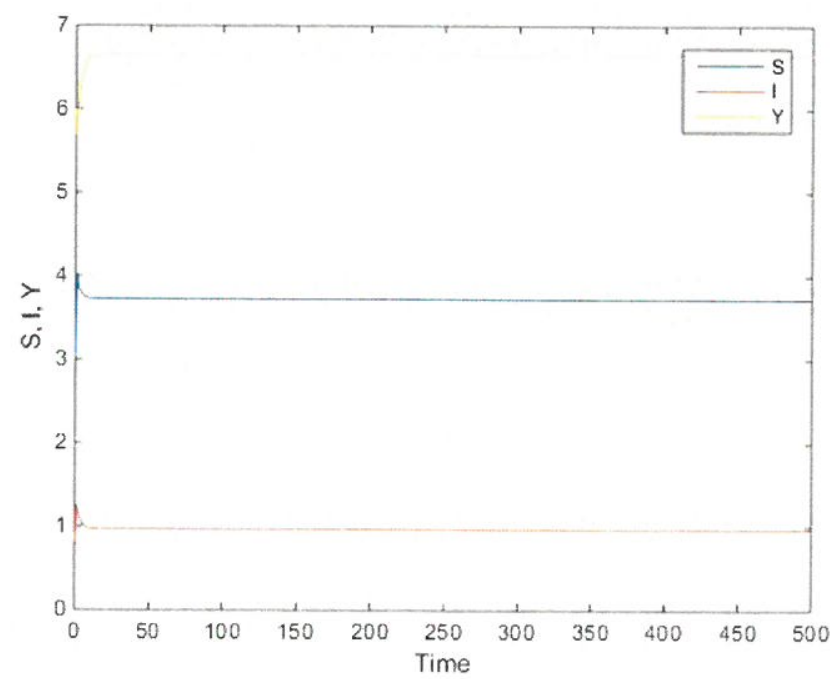

Figure 8: The solution for system (2.1) satisfying the values in Table 2 for the parameters. 


\section{Disease Control}

Proposition 7.1 If $\frac{\lambda S^{*}}{\gamma}<1$, then the disease will be eradicated from the ecosystem.

Proof. Using the second equation of system (2.1), we get

$$
\frac{d I}{d t}=I\left(\lambda S-\frac{c I Y}{S+\alpha I}-\gamma\right)<I(\lambda S-\gamma) .
$$

Thus, if $\frac{\lambda S}{\gamma}<1$, then $\frac{d I}{d t}<0$ and $I \rightarrow 0$ as $t \rightarrow \infty$. Hence ultimately there will be no infection and disease will disappear from the ecosystem. To explain it conveniently we recall the basic reproduction number $R_{0}$ defined by

$$
R_{0}=\frac{\lambda S^{*}}{\gamma} .
$$

Thus $R_{0}<1$ implies that infected prey will disappear from the ecosystem and disease will be eradicated from the prey population but $R_{0}>1$ indicates that the disease can invade or can be eradicated from the prey population.

\section{Summary and Conclusions}

We studied a prey-predator interaction model where prey are infected with a fatal disease. Predators preferentially interact with both uninfected and infected prey based on their numerical superiority. If uninfected prey are high in number then the predator will interact more with them and vice versa. Predators find it much easier to catch infected prey and, as a result, infected prey makes up a large proportion of the diet of the predator. The prey become much easier to catch after they are weakened because they come to the surface to try and increase their oxygen intake.

There are four biologically meaningful equilibria, for which we discussed their stabilities. We find some conditions under which there will be no feasible equilibria, two distinct positive equilibria, and a unique positive equilibrium. We obtained a threshold value $R_{0}$ such that $R_{0}<1$ leads to the complete disappearance of infected prey from the ecosystem and the eradication of the disease from the prey population. If the death rate or removal of infected prey is high and transmission rate from uninfected prey to infected prey is low then the chance of disease eradication from the ecosystem will be high. We have identified two important parameters which are crucial for disease eradication from the ecosystem.

\section{Acknowledgements}

We are very thankful to unknown reviewer for his valuable comments and suggestions which led to an improvement of our original manuscript. 


\section{References}

[1] Arino, O., Abdllaoui, A., El-Mikram, J. and Chattopadhyay, J., Infection on the prey population may act as a biological control in a ratio-dependent predator-prey model, Nonlinearity, 17, 1101-1116, 2004.

[2] Venturino, E, Epidemics in predator-prey model, disease in the predators, IMA Journal of Mathematics Applied in Medicine and Biology, 19, 185-205, 2002.

[3] Freedman, H.I., A model of predator-prey dynamics as modified by the action of a parasite, Mathematical Biosciences, 99, 143-155, 1990.

[4] Haque, M. and Chattophadhyay, J., Influences of a nonlinear incidence rate in an eco-epidemiological model of the Salton Sea, Nonlinear Studies, 10, 373-388, 2003.

[5] Xiao, Y. and Chen, L., Modelling and analysis of a predator-prey model with disease in the prey, Ecological Modelling, 136, 103-112, 2001.

[6] Mukhopadhyay, B. and Bhattacharyya, R., Role of predator switching in an eco-epidemiological model with disease in the prey, Ecological Modelling, 220, 931-939, 2009.

[7] Chattopadhyay, J. and Bairagi, N., Pelicans at risk in the Salton Sea - an eco-epidemiological model, Ecological Modelling, 136, 103-112., 2001.

[8] Bairagi, N, Chaudhuri, S. and Chattopadhyay, J., Harvesting as a disease control measure in an eco-epidemiological system - A theoretical study, Mathematical Biosciences, 217, 134-144, 2009.

[9] Greenhalgh, D. and Haque, M., A predator-prey model with disease in the prey species only, Mathematical Methods in the Applied Sciences, 30, 911-929, 2007.

[10] Hui, Z., Zuoliang, X. and Xin, W., Analysis of an eco-epidemiological model with time delay, Rocky Mountain Journal of Mathematics, 38, 1877-1886, 2008 .

[11] Sarkar, R. R., Chattopadhyay, J. and Bairagi, N., Effects of environmental fluctuation on an eco-epidemiological model of the Salton Sea, Environmetrics, 12, 289-300, 2001.

[12] Rocke, T., Euliss, N.H. Jr. and Samuel, M.D., Environmental characteristics associated with the occurrence of avian botulism in wetlands of a northern California refuge, Journal of Wildlife Management, 63, 358-368, 1999 .

[13] Bairagi, N., Sarkar, R. R. and Chattopadhyay, J., Impacts of an incubation delay on the dynamics of an eco-epidemiological system - a theoretical study, Bulletin of Mathematical Biology, 70, 2017-2038, 2008. 
[14] Chattopadhyay, J. and Arino, O., A predator prey model with disease in the prey, Nonlinear Analysis, 36, 747-766, 1999.

[15] Stephens, D. W. and Krebs, J. R., Foraging Theory, Princeton, New Jersey, Princeton University Press, 1986.

[16] Fisher-Piett, C.R., Soc., Biolgeogr., 92, 47-48, 1934.

[17] Renzick, D., The impact of predation on life history evolution in Trinidadian guppies: Genetic basis of observed life history, Evaluations, 36, 1236, 1982.

[18] Lawton, J. H., Beddington, J.R. and Bonser, R., Switching in invertebrate predators, Ecological Studies, 9, 144-158, 1974.

[19] Halling, C.S., Principles of insect predation, Annual Review of Entomology, 6, 163-182, 1961.

[20] Takahashi, F., Reproduction curve with two equilibrium points: a consideration of fluctuation of insect population, Researches on Population Ecology, $47,733-745,1964$

[21] May, R.M., Stability and complexity in model ecosystems, Princeton, New Jersey, Princeton University Press, 1973.

[22] May, R.M., Some mathematical problems in biology, Providence, Rhode Island, American Mathematical Society, 4, 11-29 , 1974.

[23] Tansky, M., Switching effects in prey-predator system, Journal of Theoretical Biology, 70, 263-271, 1978.

[24] Pranjneshu and Holgate, P., A prey-predator model with switching effect, Journal of Theoretical Biology, 125, 61-66, 1987.

[25] Khan, Q.J.A., Bhatt, B.S. and Jaju, R.P., Stability of a switching model with two habitats and a predator, Journal of the Physical Society of Japan, 63, 1995-2001, 1994.

[26] Khan, Q.J.A., Bhatt, B.S. and Jaju, R.P., Switching model with two habitats and a predator involving group defence, Journal of Nonlinear Mathematical Physics, 5, 212-219, 1998.

[27] Ruxton, G.D., Khan, Q.J.A. and Al-Latwatia, M., The stability of internal equilibria in predator-prey models with breeding suppression, IMA Journal of Mathematics Applied in Medicine and Biology, 19, 207-219, 2002.

[28] Khan, Q.J.A., Balakrishnan, E. and Wake, G.C., Analysis of a predatorprey system with predator switching, Bulletin of Mathematical Biology, 66, 109-123, 2004. 
[29] Lafferty, K.D and Morris, A.K, Altered behaviour of parasitized killfish increases susceptible to predation by bird final hosts, Ecology, 77, 1390, 1996.

[30] Arnold, Ordinary Differential Equations, Springer-Verlag, New York, 1971. 